\section{PREVENTIVE DENTISTRY}

\section{Effect of fluoridated milk on caries in primary teeth: 21-month results}

Bian JY, Wang WH et al. Community Dent Oral Epidemio/ 2003; 31 : 241-245

Fluoridated milk reduced caries increment by about 70\%.

In several Beijing kindergartens, 417 children were given $200 \mathrm{ml}$ milk containing $2.5 \mathrm{mg} / \mathrm{l}$ of fluoride each weekday, and $250 \mathrm{ml}$ for use each Saturday and Sunday, while 247 children at other kindergartens were control subjects. Drop out rates were respectively $22 \%$ and $19 \%$. At the start of the study, the mean age of children in both groups was 4.5 yrs. Water fluoride levels were $<0.3 \mathrm{mg} / \mathrm{l}$, and milk levels $<0.02 \mathrm{mg} / \mathrm{l}$.

Data from the 1999 national survey gave the caries prevalence in Beijing 5-yr-olds as 76\% and mean dmft as 4.2, of which $\mathrm{ft}$ was 0.5. There was good correlation between examiners, with kappa values around 0.87-0.91, although not all examiners were blind. At baseline, mean test dmft was 3.2 and control, 3.5, with respective caries prevalences $66 \%$ and 68\%. Caries was arrested in a mean of 0.3 teeth in the test group and 0.1 in controls, while 51\% of the test group, and 73\% of the control group, had new caries. Respective increments after 21 months were 0.4 and 1.3, and prevalences increased to $72 \%$ and $82 \%$. The authors note that the trial was not randomized, but that the groups were similar in all essential respects.

doi:10.1038/sj.bdj.4810823

\section{ORTHODONTICS; PERIODONTICS}

\section{Does labial movement of lower incisors} influence the level of the gingival margin? A case-control study of adult orthodontic patients

Allais D, Melsen B Eur J Orthod 2003; 25: 343-352

Recession was not significantly greater in adult patients who had received this treatment than in a matched group of controls.

Lower incisor position is an important consideration in orthodontic treatment, but there is disagreement over whether it is possible to cause gingival recession by proclination. In this study, records were compared of 150 patients who had been treated with labial movement of lower incisors and 150 control patients awaiting orthodontic treatment. Dental casts and projected slides were used to assess recession.

The two groups were matched for age (mean of each group was 34 yrs) and gender, and independent variables were found to be similar. Slides were more readable than casts, and using the former, 35\% of the test group had recession on at least one tooth, compared with $17 \%$ of the controls $(P<0.05)$. However, the mean recession of all lower incisors was $0.36 \mathrm{~mm}$ in the test group and 0.22 in controls (NS). The authors did not consider the difference to have any clinical significance.
ORTHODONTICS; PERIODONTICS

Orthodontic movement into infrabony
defects in patients with advanced
periodontal disease: a clinical and
radiological study

Corrente G, Abundo R et al. J Periodonto/ 2003; 74: 1104-1109

Orthodontic intrusion of extruded teeth, soon after periodontal surgery, gave satisfactory treatment results.

There is uncertainty over whether orthodontic treatment may have adverse effects in the periodontally involved dentition. In this cohort study, 10 patients aged 33-53 yrs, and each with one extruded maxillary central incisor with a proximal infrabony defect, were first treated nonsurgically for periodontal disease. Subsequently, flap surgery was performed, and 7-10 days later orthodontic treatment was started with light continuous forces. Periodontal maintenance was continued throughout. After a mean 10 months, permanent retention was instituted with Maryland splints.

Mean clinical attachment level in the defects improved from 9 to $3.5 \mathrm{~mm}$, and probing depth from 7 to $3 \mathrm{~mm}$. Mean radiographic bone contour improved from 4.3 to $3 \mathrm{~mm}$ vertically, and 3.4 to 2 mm horizontally. There was some measurable improvement in all individual cases. No adverse orthodontic effects were observed. The authors recommend their approach for patients with this type of problem.

\section{doi:10.1038/sj.bdj.4810825}

\section{ONCOLOGY; HEAD AND NECK SURGERY}

Influence of condition of surgical margins on local recurrence and disease-specific survival in oral and oropharyngeal cancer

McMahon J, O'Brien CJ et al. Br J Oral Maxillofac Surg 2003; 41: 224-231

Width of surgical margins did not affect subsequent complications.

Some authorities have recommended excision margins of $>1 \mathrm{~cm}$ for these tumours, but wide margins increase functional impairment. In this study 332 patients treated at 2 centres with identical protocols were followed up for a minimum 1 year (around 4/5 of recurrence is within 2 yrs). Depending on diagnostic findings, comprehensive neck dissection and/or radiotherapy was added to the basic surgical procedure aimed at $1 \mathrm{~cm}$ margins. Follow-up was $>2$ yrs for 177 patients.

Local recurrence occurred in 48 patients, nodal recurrence in 32 , and distant metastasis in 24. Univariate analysis showed a wide range of factors predicting local recurrence including the condition of excision margins, but multivariate analysis identified only the presence and extent of regional nodal involvement as an independent predictor of disease-specific survival. The authors advise $1 \mathrm{~cm}$ margins as adequate, and consider that other factors have greater effects on recurrence.

doi:10.1038/sj.bdj.4810826 